\title{
Pancreatic Diabetes after Distal Pancreatectomy: Incidence Rate and Risk Factors
}

Purpose: Pancreatectomy can impair production of endocrine and exocrine hormones. In this study, we evaluated: 1) the incidence rate of diabetes in patients undergoing distal pancreatectomy; 2) the correlation between the occurrence of pancreatic diabetes and the extent of the resected pancreas; and 3) factors associated with the development of pancreatic diabetes. Methods: We retrospectively reviewed the cases of 26 patients who could be compared in abdominal computed tomography before and after distal pancreatectomy for benign or malignant lesions between January, 1999 to June, 2010.

Results: The incidence of pancreatic diabetes was $19.2 \%$. Obese patients $\left(\mathrm{BMl}>25.0 \mathrm{~kg} / \mathrm{m}^{2}\right)$ had a higher incidence $(p=0.029)$ of pancreatic diabetes after distal pancreatectomy than non-obese patients. The diabetes group had larger volumes of resected pancreas, but the difference was not statistically significant $(p=0.105)$.

Conclusion: Several factors may be associated with the development of pancreatic diabetes after distal pancreatectomy. It is necessary to closely follow-up development of pancreatic diabetes regardless of the extent of resection.

Key Words: Pancreas, Diabetes mellitus, Postoperative diabetes, Distal pancreatectomy

\author{
Ka-Jeong Kim, M.D., Chi-Young \\ Jeong, M.D., Sang-Ho Jeong, M.D., \\ Young-Tae Ju, M.D., Eun-Jung \\ Jung, M.D., Young-Joon Lee, M.D., \\ Sang-Kyung Choi, M.D., Woo-song \\ Ha, M.D., Soon-Tae Park, M.D., \\ Soon-Chan Hong, M.D.
}

Department of Surgery, Gyeongsang National University Hospital

\section{Corresponding Author}

Soon-Chan Hong

Department of Surgery, Gyeongsang National University Hospital, 90, Chilam-dong, Jinju 660-702, Korea. Tel: +82-55-750-8096

Fax: +82-55-750-8732

E-mail: 97bluemoon@naver.com

Received: 2011. 1. 20

Accepted: 2011. 4. 16

\section{Introduction}

The pancreas is an organ that produces endocrine and exocrine hormones and plays an important role in glucose metabolism. As such, a pancreatectomy can dysregulate production of endocrine and exocrine hormones. Alpha cells, which secrete glucagon, are distributed mostly in the tail of the pancreas. PP cells, which secrete pancreatic polypeptides, are distributed in the head of the pancreas. Beta cells, which secrete insulin, are distributed throughout the entire pancreas. ${ }^{1}$ Accordingly, the resulting excision site and extent of a pancreatectomy can influence the development of hormonal abnormalities.

The exact minimum pancreatic volume for maintenance of normal blood glucose levels is not known. In some cases, when pancreatic parenchyma is normal, glucose metabolism has not changed markedly after excision of more than $80 \%$ of the pancreas.

Distal pancreatectomy is the safe standard procedure for lesions found in the body or tail of the pancreas. It is associated with relatively low mortality and morbidity rates. The aim of this study was to evaluate 1) the incidence of diabetes in patients undergoing distal pancreatectomy 2) 
the correlation between the occurrence of pancreatic diabetes and the volume of resected pancreas, and 3) factors related to the occurrence of pancreatic diabetes.

\section{Methods}

Patients $(n=56)$ undergoing distal pancreatectomy due to lesions in the body or tail of the pancreas were investigated in our institution from January, 1999 to June, 2010. We selected twenty-six patients who (1) had surgery for benign or malignant pancreatic diseases and (2) for whom comparisons could be made from abdominal computed tomography images taken before and after surgery.

Patients who had distal pancreatectomy due to trauma were excluded because combined injuries might have affected postoperative results. Patients who had diabetes before surgery were also excluded.

The amount of resected pancreas is generally estimated along the portal vein or superior mesenteric vessels. ${ }^{2,3}$ However, in this study, the extent was calculated in comparison with the volume differences before and after surgery by manually tracing each section of pancreatic area with Picture archiving and communication software (PACS)

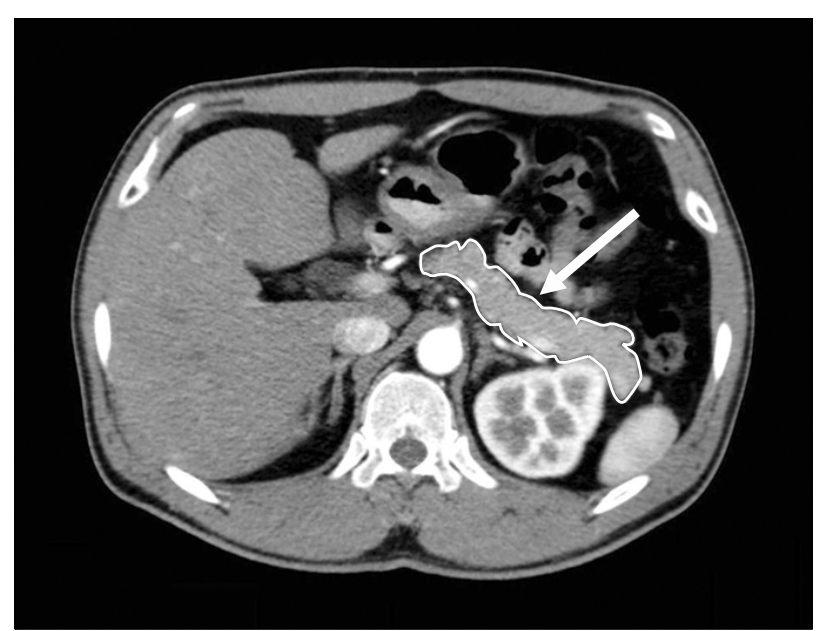

Fig. 1. Measurement of the volume of the pancreas. All pancreas areas were manually traced on every CT image section (arrow). We calculated the total volume of the pancreas and the residual volume after distal pancreatectomy. using CT images (Fig. 1).

Pancreatic diabetes was defined as a patient who had no problem with blood sugar control before pancreatectomy, but who came to need hypoglycemic agents or insulin during the follow-up period after surgery. Obesity was defined as a $\mathrm{BMI}>25.0 \mathrm{~kg} / \mathrm{m}^{2}$.

Based on the medical records of each patient, we investigated associations of time to the occurrence of pancreatic diabetes with the following variables: age, sex, medical diagnosis, name of operation, resection of other organs, operation time, blood transfusion during operation, complications after surgery, postoperative hospitalization periods, follow-up period after surgery.

\section{Statistical analysis}

Continuous, normally distributed variables are represented as mean $( \pm \mathrm{SD})$. Discontinuous variables are expressed as median (range). Continuous variables in each group were compared by an independent sample $t$ test; categorical variables by the $X^{2}$ test. All analyses were performed using SPSS statistical software for Windows, version 12.0 (SPSS Inc, Chicago, Illinois). A $p$-value $<0.05$ was considered statistically significant.

\section{Results}

\section{Patient characteristics and surgical results}

Among the twenty-six patients, nine were male, seventeen were female and the mean age was 55.9 years. Indications included seven cases of malignant tumors in the body or tail of the pancreas (26.9\%), five cases of mucinous cystic neoplasm (19.2\%), five cases of serous cystic neoplasm (19.2\%), three cases of pancreatic pseudocyst $(11.5 \%)$, three cases of intraductal papillary mucinous neoplasm $(11.5 \%)$, two cases of solitary pseudopapillary neoplasm (7.7\%) and one case of ectopic spleen which was diagnosed as neuroendocrine tumor preoperatively (3.8\%).

Among patients with distal pancreatectomy, there were thirteen cases of laparotomy $(50 \%)$ and thirteen cases of 
laparoscopy (50\%). In the cases of laparoscopic distal pancreatectomy, the splenic vessels and spleen were preserved in 9 cases $(69.2 \%)$ while resection of the spleen was done in 4 cases $(30.3 \%)$. The reasons for resection with the spleen were severe adhesion (two cases) and severe hemorrhage (two cases). The mean operation time was $310.27 \pm 122.53$ minutes and the mean postoperative hospitalization period was $17.27 \pm 8.28$ days. Complications after surgery occurred in 11 cases (42.3\%) including Pancreatic fistula (six cases), abdominal fluid collection (3 cases), fistula of resected transverse colon (1 case), and wound infection (1 case) (Table 1).

\section{Factors affecting the occurrence of pan- creatic diabetes after pancreatectomy}

There were 5 cases (19.2\%) of pancreatic diabetes during the follow-up period after distal pancreatectomy. Two

Table 1. Demographic and operative data of patients undergoing distal pancreatectomy

\begin{tabular}{lc}
\hline Age, mean \pm SD (years) & $55.9 \pm 14.89$ \\
Sex & 9 \\
Male & 17 \\
Female & \\
Indication, n (\%) & $7(26.9 \%)$ \\
Pancreatic cancer & $5(19.2 \%)$ \\
Mucinous cystic neoplasm & $5(19.2 \%)$ \\
Serous cystic neoplasm & $3(11.5 \%)$ \\
Pseudocyst & $3(11.5 \%)$ \\
Intraductal papillary mucinous neoplasm & $2(7.7 \%)$ \\
Solitary pseudopapillary neoplasm & $1(3.8 \%)$ \\
Ectopic pancreas & $310.27 \pm 122.53$ \\
Operative time, Mean \pm SD (min) & $17.27 \pm 8.28$ \\
Length of stay, mean \pm SD (days) & \\
Concomitant procedure & 16 \\
Splenectomy & 1 \\
Oophorectomy & 0 \\
Mortality & \\
Morbidity (other than pancreatic DM) & 6 \\
Pancreatic fistula & 3 \\
Intra-abdominal fluid collection & 1 \\
Wound infection & \\
Enteric fistula & \\
\hline
\end{tabular}

patients had poor glucose control right after the surgery and needed hypoglycemic agents and insulin therapy continuously. The remaining 3 patients developed pancreatic diabetes after the surgery at 5, 8, and 84 months post-operatively.

Obese patients $\left(\mathrm{BMI}>25.0 \mathrm{~kg} / \mathrm{m}^{2}\right.$ ) had a higher incidence (X vs. Y; $p=0.029$ ) of pancreatic diabetes after pancreatectomy than non-obese patients. Age $(p=0.282)$, cardiovascular basal diseases ( $p=0.463)$, benign/ malignant lesions $(p=0.173)$ or pancreatic fistula $(p=0.173)$ after surgery were not related to the development of pancreatic diabetes. Operation time was shorter in the group with pancreatic diabetes, but it was not statistically significant $(p=0.607)$.

The average volume of resected pancreas was $69.9 \%$ of the original pancreas volume in patients with pancreatic diabetes and $52.2 \%$ in patients without diabetes. The diabetes group had greater volumes of resection, but the difference was not statistically significant $(p=0.105)$. The extent was $40 \%, 68.6 \%, 73 \%, 81.5 \%$ and $86.6 \%$ in the five patients with diabetes (Table 2, 3).

\section{Discussion}

The pancreas functions as an exocrine gland that secretes various digestive juices and bicarbonate ions into the duodenum as well as an endocrine gland that secrete various hormones such as insulin, glucagon and somatostatin into the blood. The endocrine pancreas for glucose metabolism takes up $2 \%$ of the entire pancreas. It has four kinds of important secretory cells; alpha cells which secrete glucagon, beta cells which secrete insulin, delta cells which secrete somatostatin and PP cells which secrete pancreatic polypeptide. Beta cells are distributed throughout the whole pancreas while alpha cells and PP cells are locally distributed in the tail and head.

Pancreatic diabetes commonly means diabetes due to a pancreatic deficit caused by pancreatitis, trauma or pancreatectomy, but, in this study, the term is used for patients who needed hypoglycemic agents or insulin to 
Table 2. Risk factors for pancreatic diabetes mellitus

\begin{tabular}{lccc}
\hline & $\begin{array}{c}\text { Pancreatic DM }(+) \\
(\mathrm{n}=5)\end{array}$ & $\begin{array}{c}\text { Pancreatic DM }(-) \\
(\mathrm{n}=25)\end{array}$ & $p$ \\
\hline Age & $62.4 \pm 14.78$ & $54.29 \pm 14.84$ & 0.282 \\
Obesity & $3(60 \%)$ & $3(12 \%)$ & 0.029 \\
Cardiovascular disease, present & $2(40 \%)$ & $5(20 \%)$ & 0.463 \\
Malignancy & $0(0 \%)$ & $5(100 \%)$ & 0.173 \\
Pancreatic fistula, present & $0(0 \%)$ & $5(100 \%)$ & 0.173 \\
Operative time (min) & $288 \pm 96.35$ & $315.57 \pm 129.45$ & 0.607 \\
Resected pancreatic volume (\%) & $69.93 \pm 18.14$ & $52.55 \pm 20.9$ & 0.105 \\
\hline
\end{tabular}

Table 3. Five patients developed pancreatic diabetes mellitus after distal pancreatectomy

\begin{tabular}{llllcccc}
\hline & Age & Sex & Diagnosis & $\begin{array}{c}\text { BMI } \\
\left(\mathrm{kg} / \mathrm{m}^{2}\right)\end{array}$ & $\begin{array}{c}\text { Pancreas parenchymal } \\
\text { disease, present }\end{array}$ & $\begin{array}{c}\text { Resected pancreatic } \\
\text { volume }(\%)\end{array}$ & $\begin{array}{c}\text { Time to the occurrence } \\
\text { of diabetes }\end{array}$ \\
\hline Case 1 & 44 & M & Pseudocyst & 16.49 & Yes & 40 & 84 months \\
Case 2 & 60 & M & IPMN $^{\dagger}$ & 25.12 & No & 73 & 8 months \\
Case 3 & 73 & M & MCN $^{*}$ & 26.48 & No & 68.6 & 5 months \\
Case 4 & 54 & $\mathrm{~F}$ & IPMN $^{\dagger}$ & 20.12 & No & 81.5 & Immediate after surgery \\
Case 5 & 81 & M & IPMN $^{\dagger}$ & 25.56 & No & 86.6 & Immediate after surgery \\
\hline
\end{tabular}

*MCN=mucinous cystic neoplasm; ${ }^{\dagger}$ IPMN=intraductal mucinous cyst neoplasm

regulate their blood glucose after pancreatectomy.

Pancreatic diabetes is different from type I diabetes or type II diabetes. Ketoacidosis is rare in pancreatic diabetes compared with type I diabetes, and the rise of blood glucose is slight.

Pancreatic diabetes is Brittle diabetes. It differs from type II diabetes because peripheral insulin sensitivity increases and hypoglycemia is frequent when treated with insulin. The levels of serum insulin, glucagon and pancreatic polypeptide are low in pancreatic diabetes. The response of insulin is small when eating. ${ }^{1}$

Impaired glucose metabolism after pancreatectomy is known to be affected by the extent and location of the resection. The incidence of diabetes in patients who have normal pancreas parenchyma after pancreaticoduodenectomy is $10 \sim 24 \%{ }^{4-6}$; it is $8 \sim 60 \%$ in patients who have normal pancreas tissues after distal pancreatectomy. ${ }^{7,8}$

Lee et al. reported three cases of impaired glucose tolerance and ten cases of pancreatic diabetes in twentythree patients with distal pancreatectomy. In the ten patients with pancreatic diabetes, the pancreas was resected less than $40 \%$ in four patients, $40 \%$ in another four patients and $60 \%$ in two patients.

King et al. reported that the incidence of diabetes during the follow-up period was $8 \%$ after distal pancreatectomy. ${ }^{10}$ Impaired endocrine function was found in seven $(3.6 \%)$ of 197 patients after central pancreatectomy. ${ }^{11}$

In this study, the extent of resection in the 5 patients who had pancreatic diabetes was not significantly $(p=0.105)$ greater than in those who had no diabetes. In these 5 patients, the extent of resected pancreas was 40\%, 68.6\%, $73 \%, 81.5 \%$ and $86.6 \%$ of the original pancreas. Pancreases of the four patients who did not have diabetes were resected more than $80 \%$. This means that not only the extent of resection but also other factors are implicated in the occurrence of pancreatic diabetes.

In patients with chronic pancreatitis, the incidence rate of diabetes increased to $40 \%$ after pancreaticoduodenectomy ${ }^{4}$ and $85 \%$ after distal pancreatectomy. ${ }^{2}$ There were two cases of distal pancreatectomy with chronic pancreatitis; one of 
them developed diabetes after surgery. The incidence of diabetes in chronic pancreatitis may be affected by the extent or location of resection as well as endocrine disorders due to basal pancreatic diseases.

In patients who underwent pancreatectomy in the head or tail of pancreas, the incidence of pancreatic diabetes was $5 \sim 20 \%$ in the months after surgery and increased as the follow-up period became longer. ${ }^{4,5,9,12}$ In this study, two patients had poor glucose control right after surgery. Another two patients had abnormalities in glucose control five and eight months later; the last one developed the problem eighty-four months later. The mean follow-up period was 39.5 months (1.7 126.6 months). The incidence of diabetes may increase with a longer follow up duration.

There are other risk factors such as age, ${ }^{13}$ obesity, and previous metabolic disorders that affect the incidence of diabetes after pancreatectomy as well as the extent and location of resection and pancreas parenchymal diseases. ${ }^{1,2,14}$ In this study, age and cardiovascular basal diseases were not related to pancreatic diabetes, but obesity was significantly related $(p=0.029)$. Lee et al. reported that a major factor in pancreatic diabetes was abnormal insulin secretion rather than BMI or the extent of resection.

\section{Conclusion}

Several factors besides the extent and location of resection can contribute to pancreatic diabetes. Although the complication occurs infrequently, the development of pancreatic diabetes should be checked periodically during the follow-up period.

\section{References}

1. Slezak LA, Andersen DK. Pancreatic resection: effects on glucose metabolism. World J Surg 2001;25:452-460.

2. Hutchins RR, Hart RS, Pacifico M, Bradley NJ, Williamson RC. Long-term results of distal pancreatectomy for chronic pancreatitis in 90 patients. Ann Surg 2002;236:612-618.

3. Berney T, Rüdisühli T, Oberholzer J, Caulfield A, Morel P. Long-term metabolic results after pancreatic resection for severe chronic pancreatitis. Arch Surg 2000;135:1106-1111.

4. Huang JJ, Yeo CJ, Sohn TA, et al. Quality of life and outcomes after pancreaticoduodenectomy. Ann Surg 2000; 231:890-898.

5. Lemaire E, O'Toole D, Sauvanet A, Hammel P, Belghiti J, Ruszniewski P. Functional and morphological changes in the pancreatic remnant following pancreaticoduodenectomy with pancreaticogastric anastomosis. Br J Surg 2000;87:434-438.

6. Andersen HB, Baden H, Brahe NE, Burcharth F. Pancreaticoduodenectomy for periampullary adenocarcinoma. J Am Coll Surg 1994;179:545-552.

7. Lillemoe KD, Kaushal S, Cameron JL, Sohn TA, Pitt HA, Yeo CJ. Distal pancreatectomy: indications and outcomes in 235 patients. Ann Surg 1999;229:693-698.

8. Kahl S, Malfertheiner P. Exocrine and endocrine pancreatic insufficiency after pancreatic surgery. Best Pract Res Clin Gastroenterol 2004;18:947-955.

9. Lee BW, Kang HW, Heo JS, et al. Insulin secretory defect plays a major role in the development of diabetes in patients with distal pancreatectomy. Metabolism 2006;55:135-141.

10. King J, Kazanjian K, Matsumoto J, et al. Distal pancreatectomy: incidence of postoperative diabetes. $J$ Gastrointest Surg 2008;12:1548-1553.

11. Roggin KK, Rudloff U, Blumgart LH, Brennan MF. Central pancreatectomy revisited. J Gastrointest Surg 2006;10:804-812.

12. Ishikawa $\mathrm{O}$, Ohigashi $\mathrm{H}$, Eguchi $\mathrm{H}$, et al. Long-term follow-up of glucose tolerance function after pancreaticoduodenectomy: comparison between pancreaticogastrostomy and pancreaticojejunostomy. Surgery 2004;136:617-623.

13. Falconi M, Mantovani W, Crippa S, Mascetta G, Salvia R, Pederzoli P. Pancreatic insufficiency after different resections for benign tumours. Br J Surg 2008;95:85-91.

14. Najarian JS, Sutherland DE, Baumgartner D, et al. Total or near total pancreatectomy and islet autotransplantation for treatment of chronic pancreatitis. Ann Surg 1980;192:526-542. 